\title{
Development of an Artificial Intelligence Model to Guide the Management of Blood Pressure, Fluid Volume, and Dialysis Dose in End-Stage Kidney Disease Patients: Proof of Concept and First Clinical Assessment
}

\author{
Carlo Barbieri ${ }^{a}$ Isabella Cattinelli ${ }^{a}$ Luca Neri ${ }^{a}$ Flavio Maria ${ }^{a}$ Rosa Ramos ${ }^{b}$ \\ Diego Brancaccio ${ }^{a}$ Bernard Canaud $^{\mathrm{a}}$ Stefano Stuard ${ }^{a}$

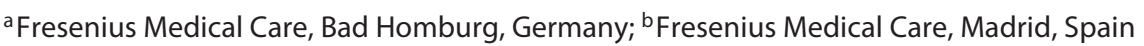

\section{Keywords}

Hemodialysis · Artificial intelligence · Intradialytic hypotension - Dialysis adequacy · Fluid overload . Hemodynamics $\cdot$ Heart rate $\cdot$ Personalized medicine . Medical decision-making

\begin{abstract}
Background: Fluid volume and blood pressure (BP) management are crucial endpoints for end-stage kidney disease patients. BP control in clinical practice mainly relies on reducing extracellular fluid volume overload by diminishing targeted postdialysis weight. This approach exposes dialysis patients to intradialytic hypotensive episodes. Summary: Both chronic hypertension and intradialytic hypotension lead to adverse long-term outcomes. Achieving the optimal trade-off between adequate fluid removal and the risk of intradialytic adverse events is a complex task in clinical practice given the multiple patient-related and dialysis-related factors affecting the hemodynamic response to treatment. State-of-the-art artificial intelligence has been adopted in other complex decision-making tasks for dialysis patients and may help personalize the multiple dialysis-related pre-
\end{abstract}

\section{KARGER}

(c) 2018 S. Karger AG, Basel

E-Mail karger@karger.com

www.karger.com/kdd scriptions affecting patients' intradialytic hemodynamics. As a proof of concept, we developed a multiple-endpoint model predicting session-specific Kt/V, fluid volume removal, heart rate, and BP based on patient characteristics, historic hemodynamic responses, and dialysis-related prescriptions. Key Messages: The accuracy and precision of this preliminary model is extremely encouraging. Such analytic tools may be used to anticipate patients' reactions through simulation so that the best strategy can be chosen based on clinical judgment or formal utility functions.

(c) 2018 S. Karger AG, Basel

\section{Introduction}

Fluid volume and blood pressure (BP) management are crucial clinical performance indicators of dialysis adequacy in end-stage kidney disease (ESKD) patients. The

Contribution from the 2nd meeting of "Science for Dialysis," organized at the University Hospital of Bellvitge, L'Hospitalet de Llobregat, Barcelona, Spain, on September 28, 2018. 
prevalence of hypertension among ESKD patients ranges between 40 and $90 \%$ according to the BP definition used, the population selected, and the timing of measurement $[1,2]$. A notable example is represented by a multicenter study including 2,535 North-American prevalent dialysis patients [1]. In this cohort, $86 \%$ of the patients had a weekly mean of predialysis systolic BP (SBP) $>150 \mathrm{~mm}$ $\mathrm{Hg}$ or a diastolic $\mathrm{BP}>86 \mathrm{~mm} \mathrm{Hg}$. Hypertension is more prevalent among ESKD patients that are initiating renal replacement therapy, since most of them present with relatively severe extracellular fluid volume expansion [3].

Clinical BP control relies mainly on reducing extracellular fluid volume overload by decreasing targeted postdialysis weight. This approach, consisting in probing "dry weight," exposes dialysis patients to intradialytic hypotensive (IDH) episodes. IDH episode is reported in 10$20 \%$ of all hemodialysis sessions, depending on the criteria, dialysis modality, or population selected. Acute volemic changes due to the ultrafiltration (UF) rate that occur during hemodialysis sessions are associated with BP and heart rate changes that contribute to repetitive hemodynamic stress and potential systemic or local ischemic insult on target organs [3].

\section{Supporting Dialysis Prescriptions with Artificial Intelligence: Rationale}

Despite significant progresses in technical aspects, fluid volume and BP control are still a challenging situation for clinicians in hemodialysis patients. On the one hand, fluid volume removal, osmolality changes, and electrolyte imbalance are well-known interacting key players of hemodynamic instability and maltolerance of hemodialysis session $[3,4]$. On the other hand, inadequate control of fluid overload and high BP may favor the occurrence of cardiovascular events and mortality. It is now established, that both chronic fluid overload and hypertension and intradialytic hemodynamic stress due to hypovolemia are associated with poor long-term patient outcomes including cardiovascular morbidity (hospitalization), altered quality of life, and mortality [5].

Fluid volume management and BP control may be addressed by diet guidance (e.g., salt restriction), pharmacological interventions (e.g., antihypertensive medications), and dialysis prescription (e.g., low dialysate sodium, longer treatment time, and hypothermic dialysis). Additionally, instrumental assessments may help tailor such prescriptions to patients' needs (e.g., body composition evaluation by bioelectrical impedance, volemia mon-

Artificial Intelligence to Optimize

Multiple Dialysis Endpoints itoring, etc.). Unfortunately, none of them has been proved individually sufficient to prevent dialysis-induced hemodynamic stress. Today, it is obvious that a combination of several of these approaches is required to achieve this primary goal and to improve the cardiovascular outcome of hemodialysis patients. Furthermore, the finetuning of such prescription is complicated by individual variations on a weekly basis and on patient's health conditions.

Reaching the optimal trade-off minimizing the risk associated with hemodynamic management while reaching dialysis treatment adequacy (i.e., Kt/V, fluid removal, etc.) is still a challenging situation for the nephrologist, given the complex interaction of all these factors. Even though the majority of ESKD patients today are in target for dialysis adequacy key indicators, such achievements may still be paid by a high rate of preventable procedurerelated hemodynamic side effects.

Artificial intelligence can be used to predict the individualized, session-specific patient reaction to dialysisrelated prescriptions on multiple relevant hemodynamic parameters (e.g., intradialytic heart rate and BP changes and trends) and dialysis adequacy parameters (e.g., Kt/V and fluid removal) so that the best strategy can be chosen based on clinical judgment or formal utility functions.

Artificial intelligence has already been successfully applied at the point of care to support physicians in the decision-making process [6-9]. The availability of accurate, longitudinal, real-life data is a key factor for the development of reproducible predictive models. The clinical system $\mathrm{EuCliD}^{\circledR}$ is an international electronic health record repository allowing point-of-care data collection of routine clinical practice information. Such detailed information includes anthropometric and sociodemographic information, results of biochemistry essays, diagnoses, medications, functional status, and dialysis access status collected by the clinical staff or automatically recorded via electronic upload of laboratory registries. Additionally, body composition and treatment data including fluid volume status, and serial monitoring of hemodynamic parameters such as BP, fluid volume and refilling rate capacity, body and dialysate temperature, fluid flow and blood flow, dialysate and plasma electrolyte concentrations as well as body weight changes are recorded for each dialysis session.

By exploiting such wealth of information, we developed a patient- and session-specific artificial neural network (ANN) to predict SBP and heart rate profiles, postdialysis body weight, and the Kt/V for each hemodialysis session. 
Table 1. Observed average dialysis-related endpoints and model prediction error

\begin{tabular}{lrrrr}
\hline & Mean & SD & Model ME & Model MAE \\
\hline Minimum SBP of the session, mm Hg & 113.0 & 23.5 & -0.16 & 9.3 \\
Postdialysis heart rate, bpm & 71.1 & 13.8 & 0.04 & 7.3 \\
Postdialysis weight, kg & 70.1 & 15.5 & 0.00 & 0.23 \\
Kt/V & 1.89 & 0.43 & 0.00 & 0.13 \\
\hline
\end{tabular}

$\mathrm{SD}$, standard deviation; ME, mean error; MAE, mean absolute error.

\section{Model Development and Validation}

An ANN [10] is a computational model based on a set of interconnected processing units organized in successive layers, loosely inspired by the neurons in the human brain, that learn to compute a specific input-output mapping by tuning a set of parameters (the weights of the connections between the units) in response to being exposed to a sufficiently large set of input and output data pairs (Fig. 1). A distinctive advantage of multilayer ANN models over traditional regression techniques is the ability to approximate any continuous function while being free of prespecified distributional assumptions. This property is particularly useful to simultaneously model multiple, heterogeneous outcomes especially when complex interactions and nonlinear effects exist between predictor variables and the outcomes of interest. Such an advantage can be achieved even with relatively simple feed-forward ANNs (that is, where connections exist only from a layer of neurons to the subsequent one) of moderate complexity and size. As no assumptions on variable distribution and form of the input-output relationship need to be imposed, the ANN approach is more flexible and more easily adapts to complex prediction problems compared to standard regression models, and even more so when compared to rule-based decision systems, which need to explicitly list a (long, and often incomplete) set of conditions and corresponding results, with no generalization capability. On the other hand, an ANN, if properly trained, can automatically infer input-output relationships from the data itself and generalize this knowledge to unseen, new data.

Our current model includes roughly 60 variables representing patient characteristics, their historical record of physiological reactions, and outcomes of previous dialysis sessions, predialysis data, and the prescribed dialysis dose for the index session. The data set used for modeling consists of 766,000 records, each representing a dialysis

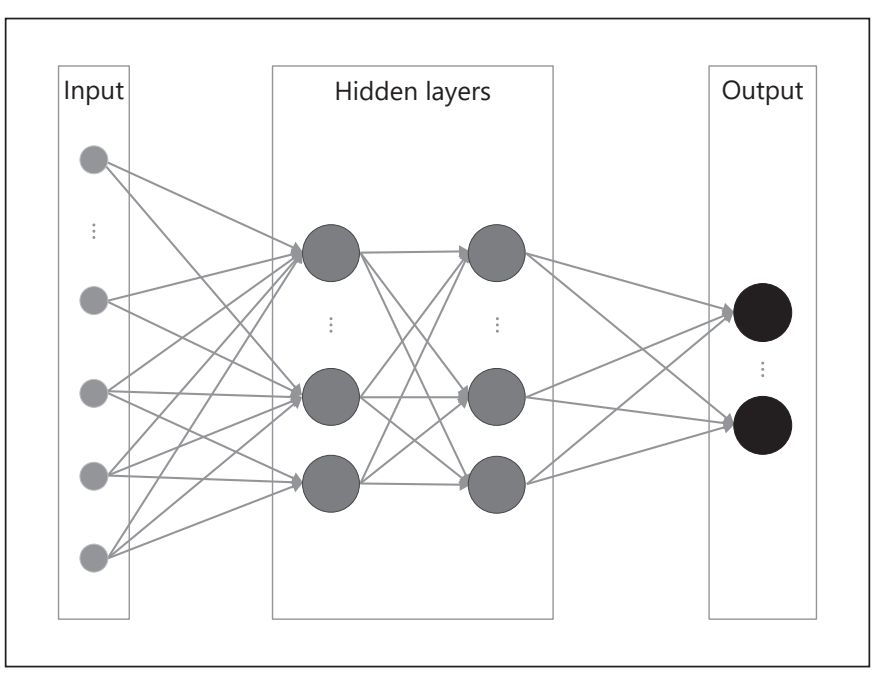

Fig. 1. Schematic depiction of a multilayer feed-forward ANN. An ANN can have an arbitrary number of inputs, hidden layers, and units in each layer. Each connection between the units has a weight value that gets tuned in response to training on input-output examples. After training, the output of the ANN can be employed to predict outcomes of interest, based on the historical data used to feed the training process.

session recorded in the Spanish NephroCare centers from January 2015 to January 2018; 80\% of the records have been used to train and validate the model (70\% for training and $10 \%$ for validation), while the remaining $20 \%$ have been used to test it after the model was finalized. The mean and standard deviation of the considered endpoints and the corresponding prediction error, expressed as the mean absolute error and mean error in the test set, are reported in Table 1.

Our preliminary results show that, while prediction of the postdialysis weight and Kt/V are virtually free of systematic error and a very small imprecision, modeling of the minimum SBP and heart rate has shown to be more difficult, possibly due to measurement errors by the ma- 


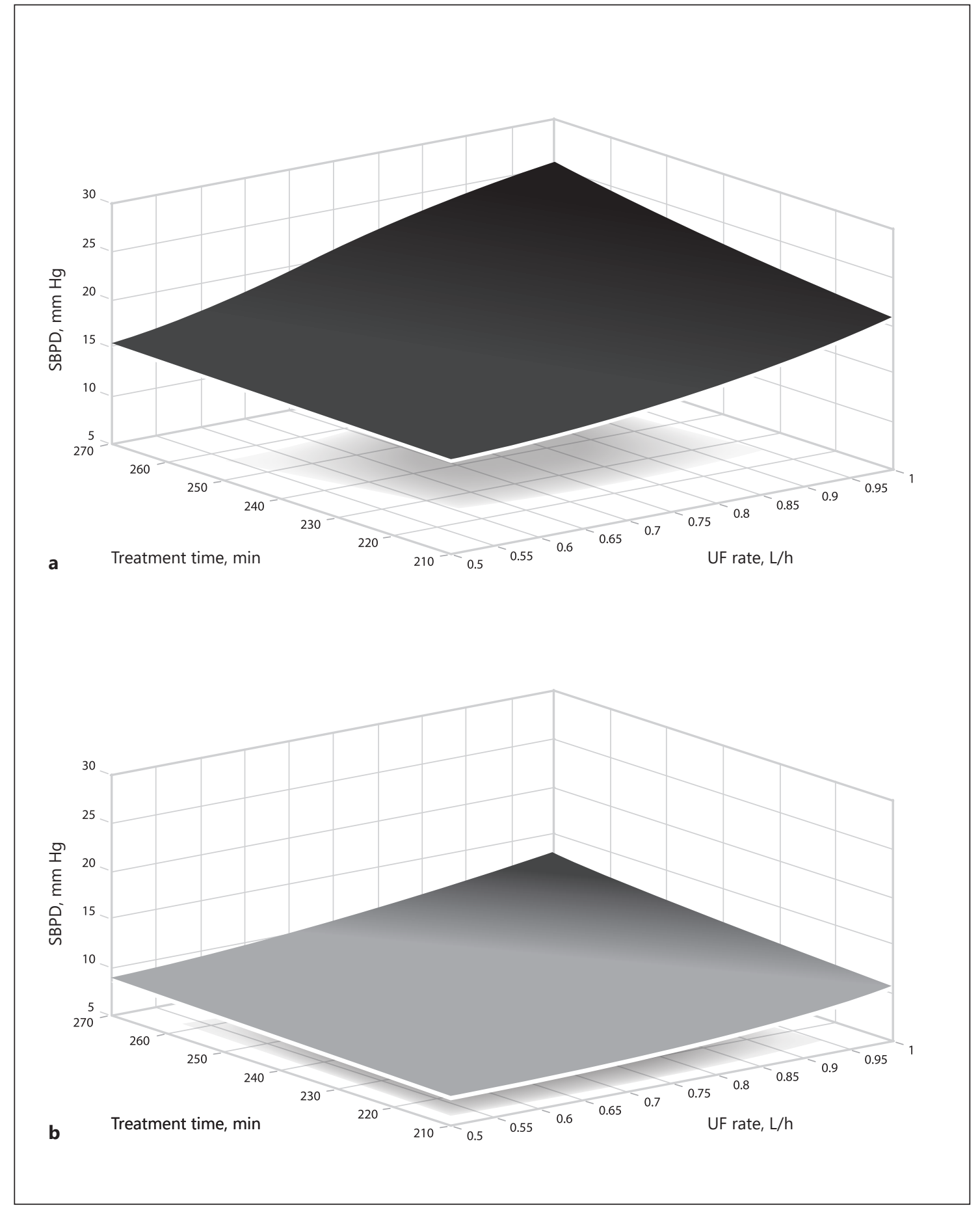

Fig. 2. Simulated intradialytic SBP change due to variation in UF prescriptions. Each point of the simulation surface represents the resulting predicted intradialytic SBP drop (SBPD) given a specific combination of UF rate and treatment time for each patient. The simulation shows that Patient A (a) and Patient B (b) have a different hemodynamic reactivity. 
Fig. 3. Optimization of UF prescription by simulated intradialytic SBP change. Each point of the simulation line represents the resulting predicted intradialytic SBP drop (SBPD) for a specific fluid removal target for Patient A. For Patients A and B, the relationship is not linear, and a steeper increase in SBPD is observed at higher UF volumes. Additionally, several equivolumetric combinations of treatment time and UF rate produce different SBPD estimates. FVL, fluid volume removal.

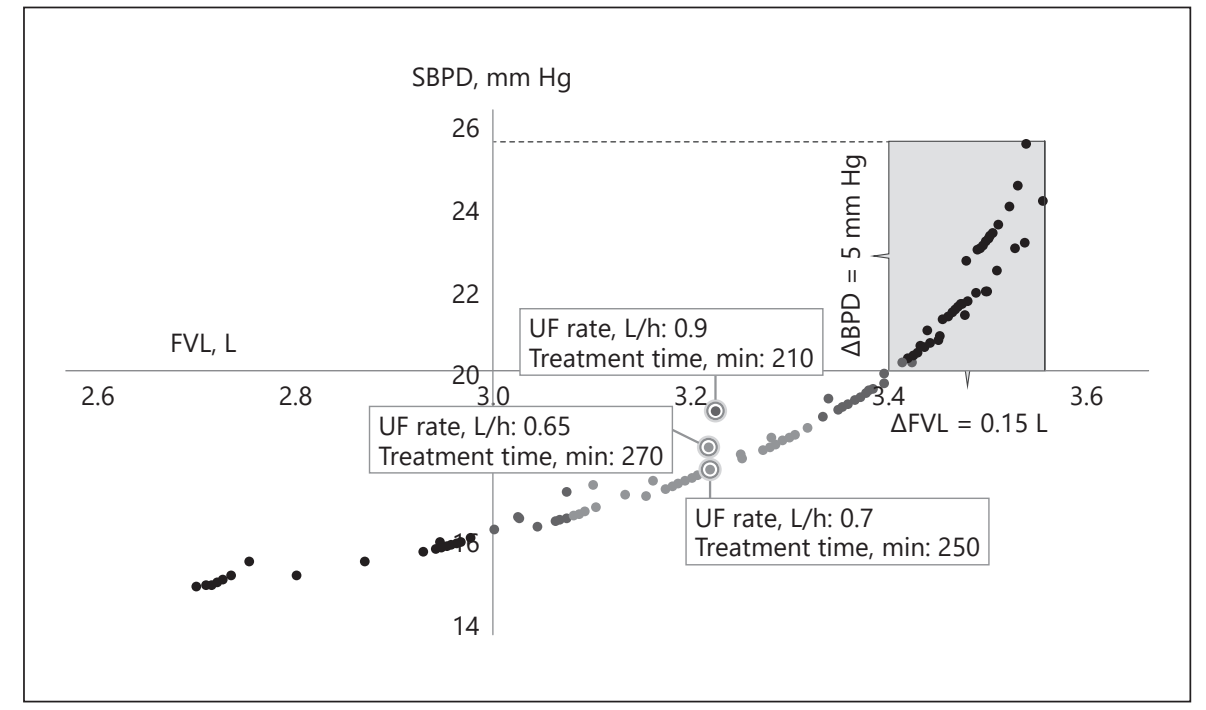

Table 2. Patient characteristics

\begin{tabular}{lcc}
\hline & Patient A & Patient B \\
\hline Weight, kg & 80 & 98 \\
Heart rate, bpm & 76 & 68 \\
DBP, mm Hg & 82 & 60 \\
Body fat mass, kg & 40 & 57 \\
Age, years & 80 & 56 \\
Diabetes vintage, years & 10 & 30 \\
HTN vintage, years & 12 & 30 \\
D-sodium, mEq/L & 140 & 138 \\
S-sodium, mEq/L & 137 & 140 \\
Ferritin & 362 & 40 \\
Hemoglobin & 10 & 13 \\
Glucose & 225 & 97 \\
Phosphate & 5 & 8 \\
\hline
\end{tabular}

DBP, diastolic BP; HTN, hypertension.

chine-integrated sensors and the naturally erratic variations in hemodynamic reaction physiology. Nevertheless, the mean absolute error shows acceptable average errors, which support the use of such a model in clinical practice. The error in SBP is less than the average white-coat effect and has presumably a small clinical significance in the context of hemodialysis patients who have SBP in the hypertensive range. Given that IDH episodes are defined by sustained and symptomatic decrease in SBPs $>30 \mathrm{~mm} \mathrm{Hg}$ or SBPs $<90 \mathrm{~mm} \mathrm{Hg}$, our model appears to be sufficiently accurate and precise to allow physicians anticipating the risk of IDH before the beginning of each session. Fur- thermore, the prediction error observed for the postdialysis heart rate model would most likely have marginal effect on our model's ability to discriminate serious tachyor bradyarrhythmias considering that they are defined as $>130$ and $<40 \mathrm{bpm}$ for several seconds, respectively.

\section{How Artificial Intelligence May Help Medical Decision-Making: A Use Case}

The potential usage of the model can be illustrated by examining and comparing 2 real-life cases. For the sake of simplicity, we represent here a decision problem defined by concurrent management of two clinical endpoints (intradialytic fluid removal and SBP change) by manipulation of two prescription parameters (treatment duration and UF rate), during a specific dialysis session. Clinical data, prescriptions, and outcomes for the $2 \mathrm{pa}$ tients have been extracted and anonymized from the EuCliD database and belong to patients who were active in the Spanish network at the time of manuscript preparation. Both Patients A and B were obese (BMI = 32), had multiple comorbidities including a long history of diabetes and hypertension, had similar predialysis SBP levels (110 vs. $115 \mathrm{~mm} \mathrm{Hg}$, respectively), estimated overhydration ( $>4 \mathrm{~L}$ for both), and received the same UF prescription (UF rate: $1 \mathrm{~L} / \mathrm{h}$; dialysis time: $240 \mathrm{~min}$ ). However, only Patient A experienced a major hypotensive event during the index session. Despite similar in many respects, these 2 patients had several divergent characteristics (Table 2). Our model helps answer questions that lay 
the basis for clinical management: were the hemodynamic reactions of both patients predictable? Would the adverse event experienced by Patient A have been preventable? By providing accurate simulations, our model facilitates session-specific prognostic reasoning and allows minimizing the risk of adverse events. Figure 2 reports the simulation of SBP variations following different UF prescriptions based on our model predictions for both patients. Model-driven simulation of the index session graphically shows that Patient A (Fig. 2a) is sensitive to hemodynamic shocks caused by variations in UF prescription, whereas Patient B (Fig. 2b) is strongly resilient to such variations. A closer look at Patient A's simulated reaction allows to detect a safer UF prescription while maximizing fluid removal (Fig. 3). The case could be further explored by assessing the impact of manipulating dialysate electrolyte compositions or the temperature on heart rate and $\mathrm{BP}$ changes so that multiple endpoints can be concurrently optimized.

In conclusion, our multiendpoint model may help formally integrate the optimization of several, interrelated treatment outcomes within a single multidimensional decision-making process substituting limited singleendpoint treatment strategies mostly used in clinical practice [10].

\section{Statement of Ethics}

The authors have no ethical conflicts to disclose.

\section{Disclosure Statement}

The authors have no conflicts of interest to declare.

\section{Author Contributions}

C.B. contributed to manuscript conception and writing, the interpretation of results, and approved the final version of the manuscript. I.C. contributed to manuscript conception, data analysis, the interpretation of results, and approved the final version of the manuscript. L.N., D.B., B.C., F.M., and S.S. contributed to manuscript conception and writing, the interpretation of results, and approved the final version of the manuscript.

\section{References}

1 Agarwal R, Nissenson AR, Batlle D, Coyne DW, Trout JR, Warnock DG. Prevalence, treatment, and control of hypertension in chronic hemodialysis patients in the United States. Am J Med. 2003 Sep;115(4):291-7.

2 Salem MM. Hypertension in the hemodialysis population: a survey of 649 patients. Am J Kidney Dis. 1995 Sep;26(3):461-8.

3 Davenport A, Cox C, Thuraisingham R. Achieving blood pressure targets during dialysis improves control but increases intradialytic hypotension. Kidney Int. 2008 Mar; 73(6):759-64.

4 Zoccali C, Tripepi G, Mallamaci F, Panuccio $\mathrm{V}$. The heart rate response pattern to dialysis hypotension in haemodialysis patients.
Nephrol Dial Transplant. 1997 Mar;12(3): 519-23.

5 Chou JA, Streja E, Nguyen DV, Rhee CM, Obi $\mathrm{Y}$, Inrig JK, et al. Intradialytic hypotension, blood pressure changes and mortality risk in incident hemodialysis patients. Nephrol Dial Transplant. 2018 Jan;33(1):149-59.

6 Barbieri C, Mari F, Stopper A, Gatti E, Escandell-Montero P, Martínez-Martínez JM, et al. A new machine learning approach for predicting the response to anemia treatment in a large cohort of End Stage Renal Disease patients undergoing dialysis. Comput Biol Med. 2015 Jun;61:56-61.

7 Barbieri C, Molina M, Ponce P, Tothova M, Cattinelli I, Ion Titapiccolo J, et al. An interna- tional observational study suggests that artificial intelligence for clinical decision support optimizes anemia management in hemodialysis patients. Kidney Int. 2016 Aug;90(2):422-9.

8 Brier ME, Gaweda AE. Artificial intelligence for optimal anemia management in end-stage renal disease. Kidney Int. 2016 Aug;90(2): 259-61.

9 Dolan JG. Multi-criteria clinical decision support: A primer on the use of multiple criteria decision making methods to promote evidence-based, patient-centered healthcare. $\mathrm{Pa}$ tient. 2010;3(4):229-48.

10 Haykin S. Neural Networks: A Comprehensive Foundation. Upper Saddle River, NJ, USA: Prentice Hall PTR; 1998. 